\title{
Superbubbles in the Magellanic Clouds
}

\author{
M. S. Oey \\ Institute of Astronomy, Madingley Road, Cambridge CB3 OHA, U.K.
}

\begin{abstract}
Superbubbles that result from the stellar winds and supernovae of $\mathrm{OB}$ associations probably play a fundamental role in the structure and energetics of the ISM in star-forming galaxies. Their influence may also dominate the relationship between the different interstellar gas phases. How do superbubbles form and evolve? How do they affect the local and global ISM? The Magellanic Clouds provide a superior opportunity to study this shell-forming activity, since both stellar content and gaseous structure can be examined in detail. Here, the results of recent studies of superbubbles in the Magellanic Clouds are reviewed.
\end{abstract}

\section{Introduction}

It is now well-established that the kinetic feedback from massive stars creates shell structures in the interstellar medium (ISM). Indeed, studies of these objects in the Magellanic Clouds provide the best empirical understanding of this phenomenon. These types of shells can be roughly classified into three categories (e.g., Chu 1996; Meaburn 1980), which are summarized in Table 1: "bubbles" and supernova remnants (SNRs), which result from stellar winds and supernovae (SNe) of individual massive stars; "superbubbles," which result from the action of a few, to hundreds of, OB star winds and SNe clustered in an OB association; and "supergiant shells," which have sizes of order $\sim 1 \mathrm{kpc}$ or more. If the supergiant shells are analogously created by massive star feedback, they must be associated with starburst phenomena having total wind and SN energies of $\gtrsim 10^{54} \mathrm{erg}$. This review will focus on recent (1990's) studies of superbubbles in the Magellanic Clouds, with also a brief look at supergiant shells.

Table 1. OB star shells.

\begin{tabular}{llcc}
\hline Type & Parent population & $\log E / \mathrm{erg}$ & $\log R / \mathrm{pc}$ \\
\hline Bubble, SNR & single O, WR & 51 & $0-1$ \\
Superbubble & OB assoc. & $52-53$ & $1-2$ \\
Supergiant shell & starburst & $\geq 54$ & $2-3$ \\
\hline \hline
\end{tabular}

How do these shells evolve? Depending on the dominant physics, there are three self-similar relations that are usually used to describe the shell evolution. In the case of a single point energy deposition, as would apply to a $\mathrm{SN}$, we have 
the traditional Sedov (1959) blastwave:

$$
R \propto(E / n)^{1 / 5} t^{2 / 5},
$$

where $R$ is the shell radius, $E$ is the input mechanical energy, $n$ is a uniform ambient density, and $t$ is elapsed time. For a constant mechanical power $L$ over an extended period, there is a standard, adiabatic evolution (Pikel'ner 1968) given by,

$$
R \propto(L / n)^{1 / 5} t^{3 / 5}
$$

This model has a double-shock structure, in which the inner shock, close to the energy source, heats the wind ejecta to temperatures of order $10^{6} \mathrm{~K}$, driving the shell growth by the thermal pressure behind the outer shock at the shell. If the hot interior is able to radiate away its energy, the inner shock will collapse upon the outer one, and the shell may assume a momentum-conserving model (Steigman et al. 1975):

$$
R \propto\left(L / n v_{\infty}\right)^{1 / 4} t^{1 / 2}
$$

where $v_{\infty}$ is the wind terminal velocity. This model describes a growth rate intermediate between those of equations 1 and 2 . There are other variations of these models; for example, an extensive review of astrophysical blastwaves is given by Ostriker \& McKee (1988).

Assuming coeval star formation in OB associations, the growth of superbubbles quickly becomes dominated by SN activity, although most of those found in $\mathrm{H} \alpha$ are extremely young objects that are still wind-dominated, $\lesssim 5 \mathrm{Myr}$ old.

\section{Evolution}

Most empirical, recent studies of superbubbles attempt to test the applicability of the standard, adiabatic model. The LMC in particular, offers a fine selection of objects, which have been used to evaluate the shell dynamics and search for enclosed hot gas.

Recent dynamical studies (Oey \& Massey 1995; Oey 1996; Mac Low et al. 1998; Oey \& Smedley 1998) of individual LMC superbubbles have found a class of objects whose observed $v / R$ is far too high for self-similar growth, in view of the stellar population present ( $v$ is the shell expansion velocity). Based on excess observed X-ray luminosities, Chu \& Mac Low (1990) and Wang \& Helfand (1991a) suggest that most of these objects suffered recent SNR impacts on the shell walls. Complex velocity structures in several objects (Rosado et al. 1990; Ambrocio-Cruz et al. 1997) are consistent with this interpretation. However, Oey \& Smedley (1998) and Mac Low et al. (1998) demonstrate that blowout from a high-density into a low-density region can perfectly mimic the shell kinematics.

Although many optical superbubbles show this high $v / R$ evidence of disruption, it is important to note that other objects have been found whose dynamics are fully consistent with self-similar growth (Oey 1996). However, all of the objects, along with the high $v / R$ objects, appear to indicate a growth rate discrepancy from the standard model, where the assumed $L / n$ is overestimated by up to an order of magnitude. It is unclear whether this is caused by an overestimate in stellar wind power, or underestimate in ambient $n$, or both. 
The search for hot gas within the superbubbles is an important test of the standard model. Several studies (Chu \& Mac Low 1990; Wang \& Helfand 1991a; Magnier et al. 1996; Mac Low et al. 1998) have found objects with X-ray emission in excess of predictions. As mentioned above, these may be caused by SNR impacts. Reassuringly, there are other superbubbles that do not show $\mathrm{X}$-ray enhancements (Chu et al. 1995), and detection limits for these remain consistent with the predicted X-ray emission. Furthermore, in all superbubbles that have been examined for C IV and Si IV absorption, these high-ionization species have been found (Chu et al. 1994), and can be attributed to an interface layer between the shell wall and hot interior.

\section{Supergiant Shells}

Supergiant shells (SGS's) are more poorly understood than superbubbles. It is possible that many of these do not originate from star formation events at all. Alternative mechanisms include impacts by high-velocity clouds (e.g., TenorioTagle et al. 1987) and gamma-ray bursters (Efremov et al. 1998; Loeb \& Perna 1998). At any rate, the evolution of SGS's is likely to differ substantially from that of superbubbles because the size scales are similar to those of galactic parameters like scale height and length.

The SGS's in the LMC have been catalogued by Meaburn (1980), and only two of these have been examined in any detail. The most well-known example is LMC-4, associated with the Constellation III stellar region. This region is a remarkably well-defined shell, $\sim 600 \mathrm{pc}$ in radius, seen in both $\mathrm{HI}$ and as a ring of optical H II regions. There have been numerous studies of LMC-4 over the last two decades; the most recent work is by Domgoergen et al. (1995) and Bomans et al. (1996) studying its gaseous properties, and by Olsen et al. (1997) and Braun et al. (1997) on the stellar content. The other LMC SGS that has been examined is LMC-2, just east of 30 Dor. Its gas properties were studied by Wang \& Helfand (1991b), Caulet \& Newell (1996), and Points et al. (1999).

\section{Superbubbles and the Global ISM}

Superbubbles, as a direct manifestation of massive star feedback, are of critical importance to the global properties of the ISM. These include ISM structure and kinematics, relationship between the different gas phases, and interstellar processes like star formation.

To gain insight on the ISM structure and kinematics caused by superbubbles, it is possible to use equation 2 to derive a size and velocity distribution for superbubbles in the ISM. This also serves as an additional test of the standard, adiabatic evolution over the full lifetimes of the superbubbles. Oey \& Clarke (1997) computed the size distribution $N(R) d R \propto R^{-\alpha} d R$ for the SMC, using the $\mathrm{H}$ II region luminosity function and assuming a constant creation rate for OB associations. The observed slope $\alpha=2.7 \pm 0.6$ for H I superbubbles (StaveleySmith et al. 1997) is in remarkable agreement with the predicted slope of $2.8 \pm 0.4$, suggesting that superbubble activity can fully explain the $\mathrm{H}$ I structure of the SMC. This can be compared to $N(R) d R$ for holes in a fractal ISM structure (Elmegreen 1997), which is being quantified for the SMC by Stanimirović et 
al. (1998). They find a volume fractal dimension $D=2.5$, translating directly to $\alpha=2.5$. It is therefore too difficult to distinguish between a superbubble and fractal ISM model, using only $N(R) d R$. Indeed, the similarity in observed $N(R) d R$ for both cases in the SMC, might be indicative of a physical relation between the two structural models.

It is also possible to test for superbubble structure in the ISM by examining the morphology of the HI holes, of which $>500$ were catalogued as shells, based on elliptical shape (Staveley-Smith et al. 1997). In addition, the velocity distribution $N(v) d v$ computed by Oey \& Clarke (1998) is also consistent with the observed shell velocities for the SMC. A superbubble velocity structure could be a dominant input to the kinematics and turbulence of the ISM.

As superbubbles expand and age, their surface brightness quickly diminishes. Hence, they could be an important component of the diffuse, warm, ionized medium (WIM). Hunter (1994) obtained emission-line spectra of superbubbles in the LMC, which do show that some of the line ratios are intermediate between those for classical H II regions and WIM detected in other galaxies. Kennicutt et al. (1995) examined the morphological structure of the WIM in the Magellanic Clouds, and also found a substantial contribution from faint superbubbles and SGS's.

Finally, it is widely thought that superbubbles can trigger renewed star formation in their shells of swept-up cool gas. Several candidates have been examined in the LMC. The clearest example is DEM 34 / N11 (Walborn \& Parker 1992; Rosado et al. 1996), and additional candidates are DEM 152 / N44 (Oey \& Massey 1995) and DEM 192 / N51 D (Oey \& Smedley 1998). As mentioned above, the SGS LMC-4 has a long history of investigation as a triggered star formation candidate (e.g., Efremov 1999).

Acknowledgments. I am grateful for an IAU Travel Grant that made possible my participation in this meeting.

\section{References}

Ambrocio-Cruz, P., Laval, A., Marcelin, M., \& Amram, P. 1997, A\&A, 319, 973

Bomans, D. J., de Boer, K. S., Koornneef, J., \& Grebel, E. K. 1996, A\&A, 313, 101

Braun, J. M., Bomans, D. J., Will, J.-M., \& de Boer, K. S. 1997, A\&A, 328, 167 Caulet, A., \& Newell, R. 1996, ApJ, 465, 205

Chu, Y.-H. 1996, in The Interplay between Massive Star Formation, the ISM, and Galaxy Evolution, eds. D. Kunth, B. Guiderdoni, M. HeydariMalayeri, \& T. X. Thuan, Gif-sur-Yvette: Editions Frontières, 201

Chu, Y.-H., \& Mac Low, M.-M. 1990, ApJ, 365, 510

Chu, Y.-H., Chang, H.-W., Su, Y.-L., \& Mac Low, M.-M. 1995, ApJ, 450, 157

Chu, Y.-H., Wakker, B., Mac Low, M.-M., García-Segura, G. 1994, AJ, 108, 1696

Domgoergen, H., Bomans, D. J., \& de Boer, K. S. 1995, A\&A, 296, 523

Efremov, Y. N. 1999, this volume

Efremov, Y. N., Elmegreen, B. G., \& Hodge, P. W. 1998, ApJ, 501, L163 
Elmegreen, B. G. 1997, ApJ, 477, 196

Hunter, D. A. 1994, AJ, 107, 565

Kennicutt, R. C., Bresolin, F., Bomans, D. J., Bothun, G. D., \& Thompson, I. B. 1995, AJ, 109, 594

Loeb, A., \& Perna, R. 1998, ApJ, 503, L35

Mac Low, M.-M., Chang, T. H., Chu, Y.-H., Points, S. D., Smith, R. C., \& Wakker, B. P. 1998, ApJ, 493, 260

Magnier, E. A., Chu, Y.-H., Points, S. D., Hwang, U., \& Smith, R. C. 1996, ApJ, 464, 829

Meaburn, J. 1980, MNRAS, 192, 365

Oey, M. S. 1996, ApJ, 467, 666

Oey, M. S., \& Clarke, C. J. 1997, MNRAS, 289, 570

Oey, M. S., \& Clarke, C. J. 1999, in Interstellar Turbulence, eds. J. Franco \& A. Carraminana, Cambridge: Cambridge Univ. Press, in press

Oey, M. S., \& Massey, P. 1995, ApJ, 452, 210

Oey, M. S., \& Smedley, S. A. 1998, AJ, 116, 1263

Olsen, K. A. G., Hodge, P. W., Wilcots, E. M., \& Pastwick, L. 1997, ApJ, 475, 545

Ostriker, J. P., \& McKee, C. F. 1988, Rev. Mod. Phys, 60, 1

Pikel'ner, S. B. 1968, Astrophys. Lett., 2, 97

Points, S. D., et al. 1999, ApJ, 518, 298

Rosado, M., et al. 1990, A\&A, 238, 315

Rosado, M., et al. 1996, A\&A, 308, 588

Sedov, L. I. 1959, Similarity and Dimensional Methods in Mechanics, New York: Academic

Stanimirović, S., Staveley-Smith, L., Dickey, J. M., Sault, R. J., \& Snowden, S. L. 1999, MNRAS, 302, 417

Staveley-Smith, L., Sault, R. J., Hatzidimitriou, D., Kesteven, M., \& McConnell, D. 1997, MNRAS, 289, 225

Steigman, G., Strittmatter, P. A., \& Williams, R. E. 1975, ApJ, 198, 575

Tenorio-Tagle, G., Franco, J., Bodenheimer, P., \& Różyczka, M. 1987, A\&A, 179,219

Walborn, N. R., \& Parker, J. W. 1992, ApJ, 399, L87

Wang, Q., \& Helfand, D. J. 1991a, ApJ, 373, 497

Wang, Q., \& Helfand, D. J. 1991b, ApJ, 379, 327

\section{Discussion}

Jan Palous: The expanding shells and supershells are rather stable to Jeans, Elmegreen, Vishniac instabilities. To get them unstable we need pre-existing clouds or shell-shell collision. (See my poster, this volume)

Oey: Thank you. Indeed, we do not yet understand the conditions for triggered star formation. 
Marc Kutner: When you look at the large scale effects of superbubbles in the MCs, can you speculate on their effect on the MW?

Oey: In our paper (Oey and Clarke 1997), we examined exactly this question. We found that if we apply our method of using the observed HII LF to derive the porosity of the Galactic ISM, we obtain a fairly small value of $\sim 0.3$. However, when we use the observed SN rate for the galaxy instead of the HII LF, we obtain a porosity value that is larger by an order of magnitude. This discrepancy is not seen in the 4 other galaxies we studied. Hence, it suggests some sort of discrepancy between the observed HII LF and SN rate in the Milky Way.

Daniel Wang: Do you think that we can distinguish supershells from supergiant shells in a more physical way? Say, supershells are produced by individual OB associations, whereas supergiant shells by a combination of multiple OB associations?

Oey: You point out an inconsistency in the definitions of the shells. It's true that we define supergiant shells by virture of their "supergiant" status. At present, we do not understand the creation and evolution of these objects, and they may originate from different and/or varying mechanisms. Multiple generations of $O B$ associations could indeed be one of these mechanisms. Until we understand the nature of the origins better, it would seem best to leave the supergiant shells as morphologically defined for now. 